\title{
Marc Kirschner
}

Harvard Medical School has launched the Initiative in Systems

Pharmacology (ISP), a new effort aimed at using systems biology approaches

to help address industry's pipeline problems. Despite the applied end goal, the programme's focus will be on developing the basic science of understanding how drugs interact with and perturb complex systems, says Marc Kirschner, Chairman of Harvard Medical School's Department of Systems Biology and sponsor of the initiative. Speaking with Asher Mullard, Kirschner outlined his vision for the ISP.

\section{Q Why launch the ISP?}

In the past the issues that caused drugs to fail were mainly off-target effects, but in many cases now toxicity problems are on-target effects and the lack of efficacy in Phase II and III trials is just unexplainable. So the question becomes: could we develop a better way of predicting whether a drug will work or have intolerable side effects?

In part, this problems stems from the fact that we rarely have a situation in which one gene can be linked to one disease and targeted by one drug. The nature of our biological system is that we have relatively few genes - say 20,000 basic core genes - that are used over and over again in different contexts. So when we investigate targets, we need to better appreciate how these function in different contexts. Moreover, there are many overlapping and redundant pathways, so we need to better understand genes not as individual elements with individual functions but within the context of the circuits in which they operate.

This approach requires not just a wiring diagram, but a quantitative wiring diagram — or a street map. If you decide you want to block traffic from one side of London to the other, first you need a map so that you can see whether the road you want to block really goes from point A to point B. You also need to know about all the parallel roads that can be taken. And, you need to know the difference between a tiny alley and a major thoroughfare. We want to build such quantitative maps - and the science of developing these maps - in the hopes that they will enable researchers to better predict how drugs will act.

Q What type of projects will you undertake? Our effort has already started to some degree. We have people who are looking at how antibiotics can synergize and antagonize each other, in the hopes of developing strategies for identifying antibiotic drug combinations. Other groups are looking at quantitatively assessing cell death pathways, and at understanding how cytotoxic anticancer drugs act differently in Petri dishes versus in xenograft mice.

We will also try to understand why drugs fail. The pharmaceutical industry is interesting in that it doesn't explore its own failures. We may be able to study abandoned candidates, or related small molecules, to better understand their effects and why they failed. We need this kind of investigation, rather than the current approach of having a hunch, placing a bet and, if the horse doesn't win, coming back next week to bet on another race.

Overall, I hope that the initiative will be very diversified. We will have a few groups working on a few projects that will have loose connections with each other, but they will be linked by an interest in how drugs perturb complex systems.

\section{Q Do you expect to partner with industry} to put their experimental candidates through the systems pharmacology paces?

I would be disappointed if that were the major way in which we interacted with industry. What we need is a fundamental science that looks at how drugs interact with complex systems, and not a service facility for companies to use as a part of their drug discovery efforts.

\section{Q Why do this within academia?}

In the past decade or so, the US National Institutes of Health has pushed for more translational research efforts. One form that this has taken has been for academic institutions and medical schools to build their own drug discovery teams. This has had some good effects. But I don't think it's the best way to spend the money, because it doesn't take into account what drug companies are already good at: making drugs, screening for drugs, medicinal chemistry and more. These aspects of drug discovery cannot be adequately reproduced in an academic environment. Industry is not good, however, at identifying where to attack a disease or at predicting the global effects of inhibiting an enzyme. In academia we're not good at this either, but we study those pathways in great detail. By further developing this science, we can have the biggest impact.

I don't consider this to be an applied industrial activity either, but instead a valid area for basic science. Science in general has gained a lot from being forced to think about practical problems, and abstract or general principles can emerge from such work. When we started studying steam engines, we ended up with thermodynamics. Similarly, we might learn some things here that will help the drug pipeline, but we will also learn a great deal about how organisms function.

\section{Q What is your greatest challenge?} If I go by historical precedents, I think that we will be able to make some notable contributions in the short run. But if the community is looking for specific contributions - a cure for a specific disease, for example - then we may not be able to deliver for many years. So, our challenge is in managing expectations. If these are too specific, then we are almost guaranteed to fail. If the expectations are general, then we have much better odds of success. 